\title{
A Role for the Default Mode Network in the Bases of Disorders of Consciousness
}

\author{
Davinia Fernández-Espejo, PhD, ${ }^{1,2,3}$ Andrea Soddu, $\mathrm{PhD},{ }^{4}$ Damian Cruse, $\mathrm{PhD},{ }^{1}$ \\ Eva M. Palacios, MSc, ${ }^{2}$ Carme Junque, $\mathrm{PhD}^{2}$ Audrey Vanhaudenhuyse, $\mathrm{PhD}^{4}$ \\ Eva Rivas, MD, ${ }^{5}$ Virginia Newcombe, MD, PhD, ${ }^{3,6}$ David K. Menon, MD, PhD,, 3 \\ John D. Pickard, FMedSci, MChir, FRCS, 3,7 Steven Laureys, MD, PhD, ${ }^{4}$ \\ and Adrian M. Owen, $\mathrm{PhD}^{1}$
}

\begin{abstract}
Objective: Functional connectivity in the default mode network (DMN) is known to be reduced in patients with disorders of consciousness, to a different extent depending on their clinical severity. Nevertheless, the integrity of the structural architecture supporting this network and its relation with the exhibited functional disconnections are very poorly understood. We investigated the structural connectivity and white matter integrity of the DMN in patients with disorders of consciousness of varying clinical severity.

Methods: Fifty-two patients-19 in a vegetative state (VS), 27 in a minimally conscious state (MCS), and 6 emerging from a minimally conscious state (EMCS) - and 23 healthy volunteers participated in the study. Structural connectivity was assessed by means of probabilistic tractography, and the integrity of the resulting fibers was characterized by their mean fractional anisotropy values.

Results: Patients showed significant impairments in all of the pathways connecting cortical regions within this network, as well as the pathway connecting the posterior cingulate cortex/precuneus with the thalamus, relative to the healthy volunteers. Moreover, the structural integrity of this pathway, as well as that of those connecting the posterior areas of the network, was correlated with the patients' behavioral signs for awareness, being higher in EMCS patients than those in the upper and lower ranges of the MCS patients, and lowest in VS patients.

Interpretation: These results provide a possible neural substrate for the functional disconnection previously described in these patients, and reinforce the importance of the DMN in the genesis of awareness and the neural bases of its disorders.
\end{abstract}

ANN NEUROL 2012;72:335-343

$\mathrm{P}$

atients with disorders of consciousness (DOC) show metabolic impairments and functional disconnections within corticocortical and thalamic-cortical areas of the default mode network $(\mathrm{DMN})^{1-4}$ to an extent that appears to correspond to clinical severity. ${ }^{5}$ Thus, poorer functional connectivity is observed in vegetative state (VS) patients (who show no behavioral signs of awareness) ${ }^{6}$ than in minimally conscious state (MCS) patients (who show intermittent behavioral signs of awareness).
It is generally assumed that functional connectivity within intrinsic networks reflects structural connectivity. A plausible hypothesis, then, is that the reduced functional connectivity observed in the DMN of DOC patients reflects structural disconnections within this network, providing anatomical support for the description of these patients as suffering from "disconnection syndromes." ${ }^{\prime 8}$ However, the relationship between structure and function in the DMN is not straightforward. ${ }^{9}$ It has

View this article online at wileyonlinelibrary.com. DOI: 10.1002/ana.23635

Received Jan 1, 2012, and in revised form Apr 12, 2012. Accepted for publication Apr 20, 2012.

Address correspondence to Dr Fernández-Espejo, Brain and Mind Institute, Natural Sciences Centre, University of Western Ontario, London, Ontario, N6A 5B7, Canada. E-mail: despejo@uwo.ca

From the ${ }^{1}$ Brain and Mind Institute, University of Western Ontario, London, Ontario, Canada; ${ }^{2}$ Department of Psychiatry and Clinical Psychobiology, IDIBAPS, University of Barcelona, Barcelona, Spain; ${ }^{3}$ Wolfson Brain Imaging Centre, Department of Clinical Neurosciences, University of Cambridge, Cambridge, United Kingdom; ${ }^{4}$ Coma Science Group, Cyclotron Research Centre and Neurology Department, University and University Hospital of Liège, Liège, Belgium; ${ }^{5}$ Anesthesiology Department, Hospital Clinic, University of Barcelona, Barcelona, Spain; ${ }^{6}$ Division of Anaesthesia, Department of Medicine, University of Cambridge, Cambridge,United Kingdom; and ${ }^{7}$ Division of Academic Neurosurgery, Addenbrooke's Hospital, University of Cambridge, Cambridge, United Kingdom. 
been shown previously that some DOC patients exhibit functional impairments in the DMN, whereas the global white matter skeleton is relatively preserved. ${ }^{10,11}$

Determining whether it is the DMN that is specifically structurally disconnected in this population, or whether this functional deficit reflects more widespread white matter damage, will not only provide new information about the neuropathological underpinnings of these complex conditions, but will also shed new light on those processes that support normal awareness in healthy individuals. Here, we used probabilistic diffusion-based tractography to assess the structural integrity of the DMN in a large group of VS and MCS patients with different types of severe brain injury.

\section{Subjects and Methods \\ Participants}

A retrospective convenience sample of 81 severely brain-injured patients of varying etiologies, who met the criteria defining VS, ${ }^{6}$ MCS, ${ }^{7}$ or emerging from a minimally conscious state (EMCS), ${ }^{7}$ were assessed at 3 European centers: Addenbrooke's Hospital (Cambridge, UK), Hospital Clínic (Barcelona, Spain), and University Hospital of Liège (Belgium). A small subset of the patients included in this cohort have been described previously. ${ }^{12}$ From these, 52 patients (19 VS, 27 MCS, and 6 EMCS) met the magnetic resonance imaging (MRI) data quality criteria (Supplementary Materials) and were included in the analyses. Clinical examination was performed using the Coma Recovery Scale-Revised (CRS-R). ${ }^{13}$ The highest score achieved by each patient across the different examinations is included in the Supplementary Table. There were no significant differences between the included and excluded patients in terms of age, months postictus, gender, etiology, diagnostic distributions, or global CRS-R score. Demographic and clinical characteristics of the patients are summarized in Table 1. To account for the wide heterogeneity of behaviors that characterizes MCS patients, ${ }^{7}$ we subcategorized them into MCS- (patients who show only nonreflex responses such as localizing noxious stimuli or visually pursuing a moving or salient stimulus) and MCS+ (patients who show intelligible verbalization, yes/no responses, or command-following). ${ }^{14}$

A group of 23 age-matched controls was also recruited between the 3 participant centers. Each recruitment center contributed the same proportion of healthy controls and patients.

Informed assent was obtained from all patients' families and medical teams. All healthy participants gave informed consent. Ethical approval for the study was provided by the National Research Ethics Service (National Health Service; Cambridge), the ethics committee of the Hospital Clínic (Barcelona), and the ethics committee of the Faculty of Medicine of the University of Liège.

\section{MRI Acquisition}

For all subjects, diffusion-weighted images were acquired in $3 \mathrm{~T}$ MRI Magneton Trio Tim scanners (Siemens, Erlangen,

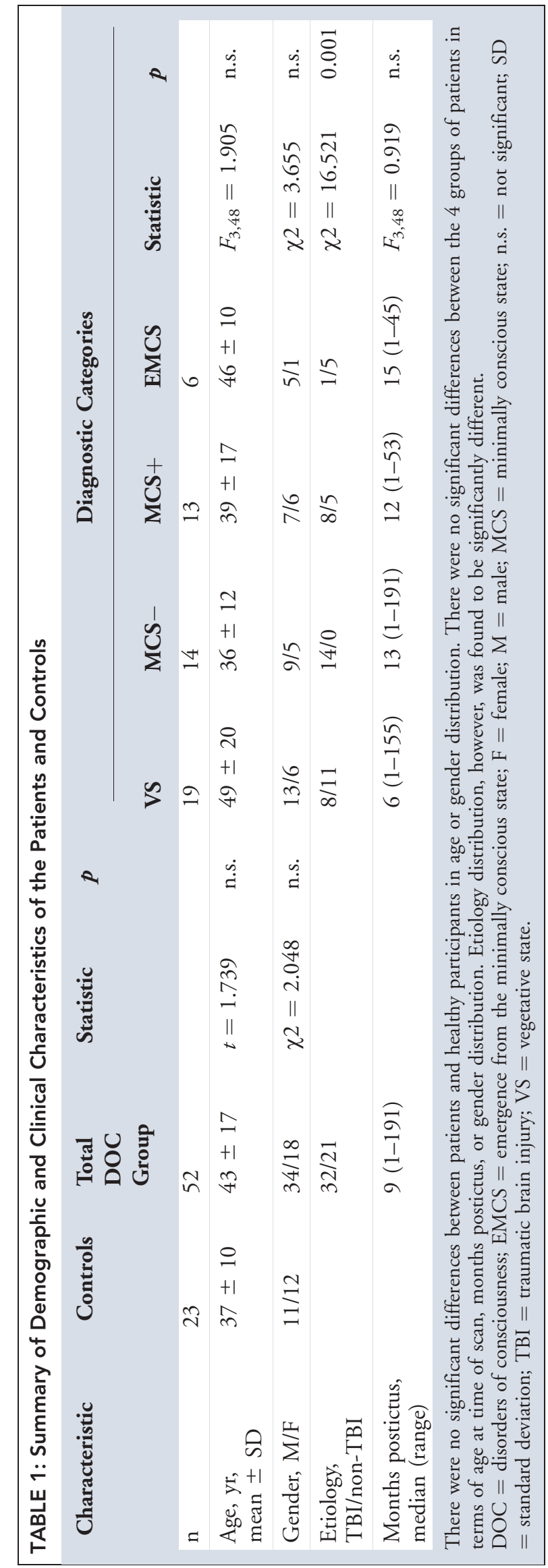




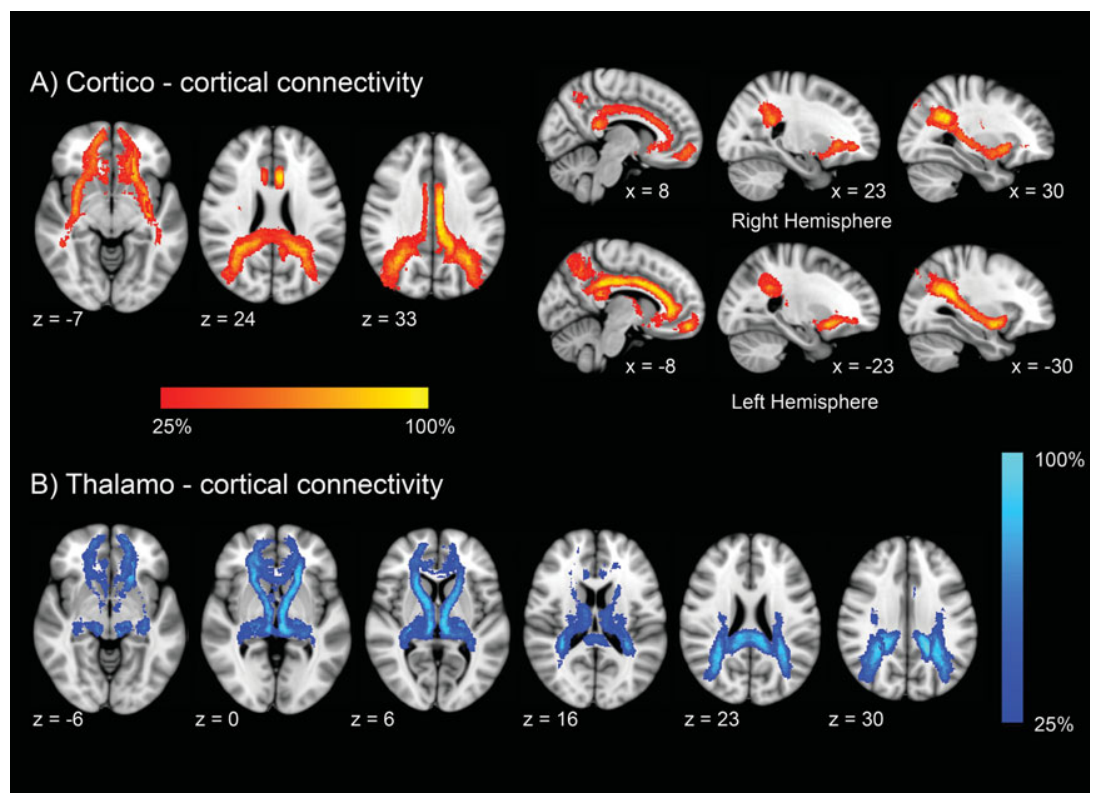

FIGURE 1: Group probability maps of reconstructed tracts in the healthy participants. Maps are thresholded at presence in at least $\mathbf{2 5 \%}$ of the subjects. Images are displayed in Montreal Neurological Institute standard stereotaxic space, and coordinates are provided for each slice.

Germany) and included diffusion sensitizing gradients applied along 12 noncollinear directions using 5 b-values ranging from 340 to $1,590 \mathrm{~s} / \mathrm{mm}^{2}$ and $5 \mathrm{~b}=0$ images (repetition time [TR] $=8,300$ milliseconds, echo time $[\mathrm{TE}]=98$ milliseconds, matrix size $=96 \times 96,63$ slices, slice thickness $=2 \mathrm{~mm}$, no gap) at the Wolfson Brain Imaging Centre (Cambridge), 30 noncollinear directions with a b-value $=1,000 \mathrm{~s} / \mathrm{mm}^{2}$ and $1 \mathrm{~b}=0$ image $(\mathrm{TR}=5,600$ milliseconds, $\mathrm{TE}=89$ milliseconds, matrix size $=122 \times 122,49$ slices, slice thickness $=1 \mathrm{~mm}$, gap $=$ $0.6 \mathrm{~mm}$ ) at the Center for Image Diagnosis (Barcelona), and 64 noncollinear directions using a b-value $=1,000 \mathrm{~s} / \mathrm{mm}^{2}$ and $2 \mathrm{~b}$ $=0$ images $(\mathrm{TR}=5,700$ milliseconds, $\mathrm{TE}=87$ milliseconds, matrix size $=128 \times 128,45$ slices, slice thickness $=3 \mathrm{~mm}$, gap $=0.3 \mathrm{~mm})$ at the University Hospital of Liège.

\section{Diffusion Tensor Imaging Data Analysis}

Data preprocessing and analysis were performed using the FSL Diffusion Toolbox (http://www.fmrib.ox.ac.uk/fsl). Fiber tracking was estimated for each subject between a pair of regions of interest (ROIs) in native space. ${ }^{15}$ Cortical ROI masks were defined in Montreal Neurological Institute standard space as $12 \mathrm{~mm}$ radius spheres centered in the 4 most significant foci described for the $\mathrm{DMN}^{16}$-medial prefrontal cortex (MPF), posterior cingulate cortex/precuneus $(\mathrm{PCC} / \mathrm{PCu})$, and both left and right temporoparietal junctions (TPJ) — and then unwarped to native space for each subject using FLIRT. ${ }^{17}$ Thalamic masks were defined as described elsewhere. ${ }^{12}$ Finally, mean fractional anisotropy (FA) values for the obtained paths connecting each pair of ROIs were calculated and used to quantify and compare the integrity of the identified paths. To assess and control for the effects of global white matter damage, we calculated the mean FA value from a white matter mask generated by applying a threshold of 0.2 to the FA map. ${ }^{18}$

\section{Statistical Analysis}

Statistical analysis was performed using PASW Statistics 18. The distribution of the dependent measures (FA mean values) was tested for normality using the Kolmogorov-Smirnov test. Group comparisons were performed using 1-way analysis of covariance (ANCOVA) for nonlateralized connections (ie, PCC/ $\mathrm{PCu}$ - MPF and TPJ left - right), and 1-way repeated measures ANCOVA, using hemisphere as the repeated measures factor, for those involving lateralized left and right paths. Group served as the between-subjects factor, and patient assessment center was used as statistical covariate in all cases. Etiology and global FA were also used as statistical covariates when appropriate. Significance was set at a $p<0.05$.

First, all 52 patients were compared with the healthy volunteers. The effect of diagnostic category on the structural integrity of the network was then investigated. Finally, to further assess the relationship between the integrity of the fibers and the clinical severity of the DOC, correlations were performed between the integrity of the fibers and the behavioral signs of awareness as indexed by the diagnoses and the associated CRS$\mathrm{R}$ scores. For the correlation analyses on lateralized paths, left and right measures were combined into a single value. Because an a priori hypothesis was defined, significance was set at a 1 -tailed $p<0.05$. Graphs were produced using SigmaPlot 10 .

\section{Results}

\section{Corticocortical and Thalamocortical Connectivity}

Fiber tracts identified in the control group can be seen in Figure 1. For detailed information about the fibers, see the Supplementary Materials. 


\begin{tabular}{|c|c|c|c|c|c|}
\hline Tractography Paths & Controls & DOC Patients & $F$ & $d f$ & $p$ \\
\hline TPJ left - right & $0.398(0.013)$ & $0.338(0.013)$ & 5.405 & 1,46 & 0.025 \\
\hline $\mathrm{PCC} / \mathrm{PCu}-\mathrm{MPF}$ & $0.382(0.010)$ & $0.344(0.007)$ & 7.522 & 1,56 & 0.008 \\
\hline MPF - TPJ & $0.395(0.012) / 0.390(0.012)$ & $0.356(0.011) / 0.347(0.011)$ & 10.363 & 1,31 & 0.003 \\
\hline $\mathrm{PCC} / \mathrm{PCu}-\mathrm{TPJ}$ & $0.365(0.015) / 0.414(0.017)$ & $0.320(0.009) / 0.334(0.010)$ & 12.407 & 1,68 & 0.001 \\
\hline $\mathrm{PCC} / \mathrm{PCu}$ - thalami & $0.417(0.014) / 0.455(0.013)$ & $0.388(0.009) / 0.399(0.009)$ & 7.205 & 1,63 & 0.009 \\
\hline MPF - thalami & $0.362(0.010) / 0.355(0.012)$ & $0.354(0.008) / 0.365(0.010)$ & 0.008 & 1,52 & 0.931 \\
\hline TPJ - thalami & $0.392(0.011) / 0.385(0.011)$ & $0.374(0.008) / 0.380(0.008)$ & 1.135 & 1,58 & 0.291 \\
\hline
\end{tabular}

Values are the predicted marginal means adjusted for center and global white matter fractional anisotropy effects. There was a main effect of group on all the corticocortical pathways as well as in the connection between PCC/PCu and thalami. Values in parentheses are standard errors. For lateralized pathways, left/right values are provided.

$\mathrm{DOC}=$ disorders of consciousness; MPF = medial prefrontal cortex; $\mathrm{PCC} / \mathrm{PCu}=$ posterior cingulate cortex/precuneus; TPJ = temporoparietal junctions.

\section{Differences between Patients and Healthy Participants}

A comparison between DOC patients and healthy participants was performed on the mean FA values extracted from the bundles connecting each pair of ROIs. For the global white matter FA, the ANCOVA (using center as covariate) revealed very significant differences between patients $(0.37 \pm 0.003)$ and healthy participants $(0.40$ $\left.\pm 0.004 ; F_{1,72}=51.642, p<0.001\right)$. As the main goal of this study was to assess the specific integrity of the $\mathrm{DMN}$, we also included global white matter FA as a covariate in the analysis of the individual pathways to control for the effect of widespread white matter damage. These ANCOVAs revealed significant FA reductions in the patients when compared to the healthy volunteers in all corticocortical pathways, as well as that connecting PCC/PCu with thalami (see Table 2 and Fig 2). For all lateralized paths, neither the effect of hemisphere nor the group by hemisphere interaction was statistically significant.

\section{Differences across Levels of Clinical Severity}

The distribution of diagnostic categories between the 3 centers was not significantly different (chi-square $=$ 5.5788, $p>0.05$ ). There were no significant differences either between the 4 groups of patients in terms of age at time of scan, months postictus, gender distribution, or global white matter FA. Etiology distribution was found to be significantly different and was therefore included as a nuisance covariate in the group comparisons. Moreover, additional ANCOVAs testing the effect of etiology on FA values returned no significant results $(p>0.25$ in all cases).
The ANCOVAs, which used academic center and etiology as covariates, revealed a significant main effect of the diagnostic category for the pathways connecting $\mathrm{PCC} / \mathrm{PCu}$ with TPJ $\left(F_{3,43}=3.894, p=0.015\right)$ and PCC/PCu with thalami $\left(F_{3,38}=6.773, p=0.001\right)$. Post hoc pair-wise comparisons on PCC/PCu-TPJ revealed that, although there was a trend for a reduction in $\mathrm{FA}$ alongside a reduction in the presence of behavioral signs of awareness (EMCS: $0.35 \pm 0.05 / 0.40 \pm 0.07$; MCS+: $0.32 \pm 0.10 / 0.31 \pm 0.07$; MCS-: $0.32 \pm$ $0.07 / 0.29 \pm 0.05$; VS: $0.26 \pm 0.07 / 0.29 \pm 0.08$ ), the effect was mainly driven by differences between VS and EMCS $\left(F_{1,21}=8.842, p=0.007\right)$. For PCC $/ \mathrm{PCu}-$ thalami, conversely, there was a more consistent trend for decreases in FA as the clinical severity of the DOC increased, with the FA values decreasing from EMCS $(0.45 \pm 0.07 / 0.47 \pm 0.06)$, to MCS $+(0.38 \pm 0.06 /$ $\left.0.40 \pm 0.07 ; F_{1,12}=10.553, p=0.007\right)$, from MCS + to MCS $-\left(0.37 \pm 0.07 / 0.36 \pm 0.06 ; F_{1,19}=5.365, p\right.$ $=0.032$, and finally, although nonsignificantly, from MCS - to VS $\left(0.35 \pm 0.08 / 0.36 \pm .07 ; F_{1,24}=\right.$ 0.760, $p>0.05$; Fig 3).

Partial correlations controlling for the same nuisance variables confirmed this positive relationship between the level of clinical severity (ie, diagnosis) and the integrity of these 2 pathways (PCC/PCu-TPJ: $r=$ 0.474, $p=0.001$; PCC/PCu-thalami: $r=0.436, p=$ $0.001)$.

\section{Correlations with CRS-R Score}

Spearman correlations were performed between CRS-R scores and mean FA values in all the reconstructed pathways as well as in the global white matter mask. CRS-R 


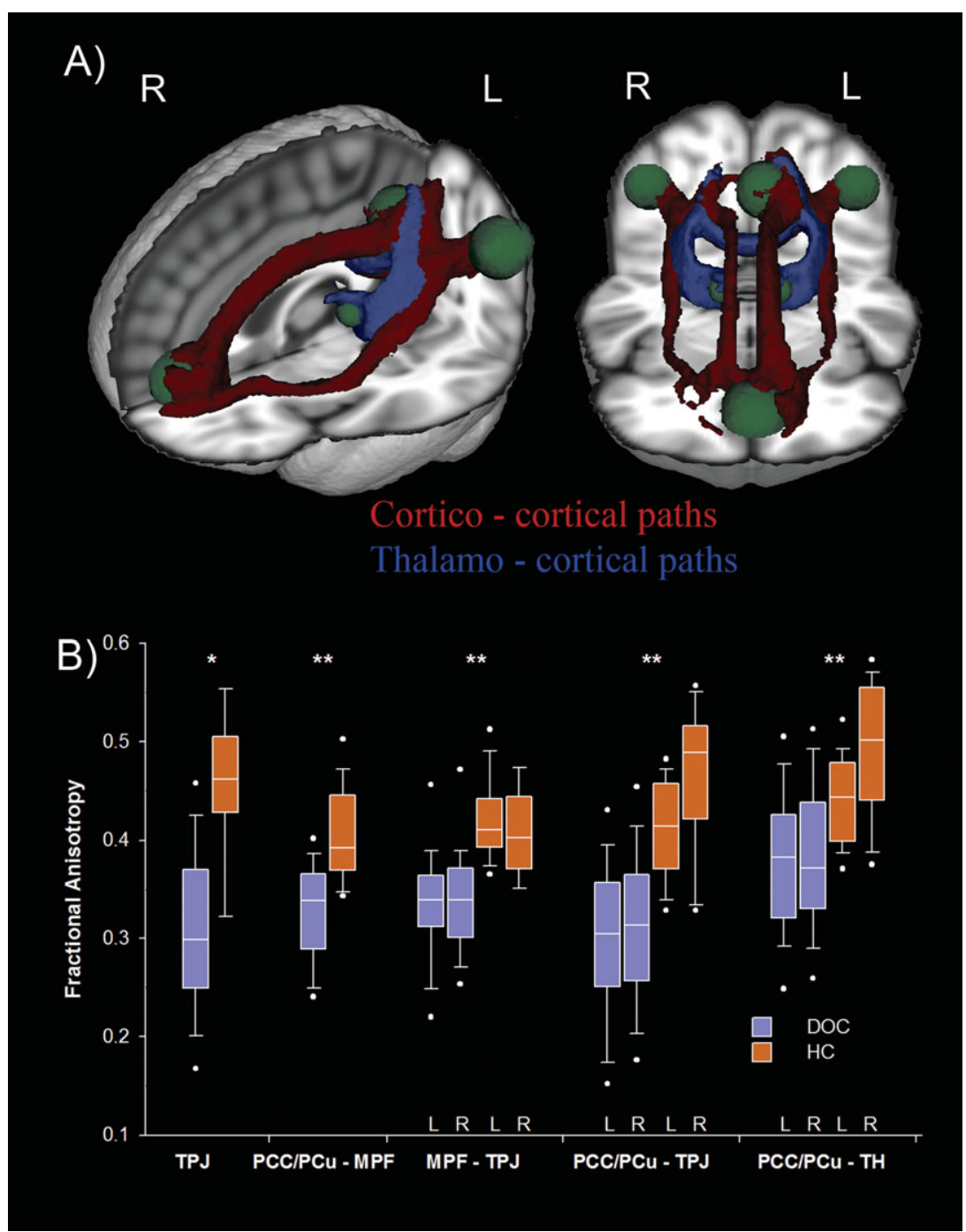

FIGURE 2: (A) Three-dimensional representation of the corticocortical (in red) and thalamocortical (in blue) paths showing reduced fractional anisotropy values in the patients relative to healthy participants. Green spheres illustrate the regions of interest used in the tractography analysis. (B) Box plots of the fractional anisotropy values from the tracts represented in A for the patients (in purple) and the healthy controls (in orange). For the lateralized paths (ie, medial prefrontal cortex [MPF] - temporoparietal junctions [TPJ], posterior cingulate cortex/precuneus [PCC/PCu] - TPJ, and PCC/PCu - thalami [TH]) values for the left (L) and right (R) hemispheres are shown. The central lines in the boxes denote the median values; the lower and upper edges represent the 25th and 75th percentiles, respectively; the error bars represent the 10th and 90th percentiles, respectively; and the closed circles represent the 5 th and 95 th percentiles. ${ }^{\star} p<0.05 ;{ }^{\star \star} p<0.01$. DOC $=$ disorders of consciousness group; $\mathrm{HC}=$ healthy participants group.

scores were significantly correlated with FA in $\mathrm{PCC} /$ $\mathrm{PCu}$-thalami (rho $=0.363, p=0.008), \mathrm{PCC} / \mathrm{PCu}-\mathrm{TPJ}$ (rho $=0.343, p=0.008)$, TPJ-thalami (rho $=0.340, p$ $=0.017$ ), and global FA (rho $=0.322, p=0.01$ )

\section{Discussion}

To our knowledge, this is the first study to assess the structural integrity of the DMN in DOC patients. Using probabilistic diffusion-based tractography, the thalamocortical and corticocortical pathways connecting the main nodes of the DMN were reconstructed, and a marked impairment of this network in DOC relative to healthy controls was observed. Moreover, this deficit correlated with the clinical severity of the disorder.

\section{DMN Structural Connectivity in the Healthy Brain}

The corticocortical tracts that were identified (ie, cingulum bundles, superior longitudinal fasciculus, inferior fronto-occipital fasciculus, and corpus callosum) are consistent with those identified in previous studies of healthy volunteers of varying ages, and from clinical groups. ${ }^{19-22}$ Interestingly, reliable connections were also found between the cortical areas of the DMN and the 

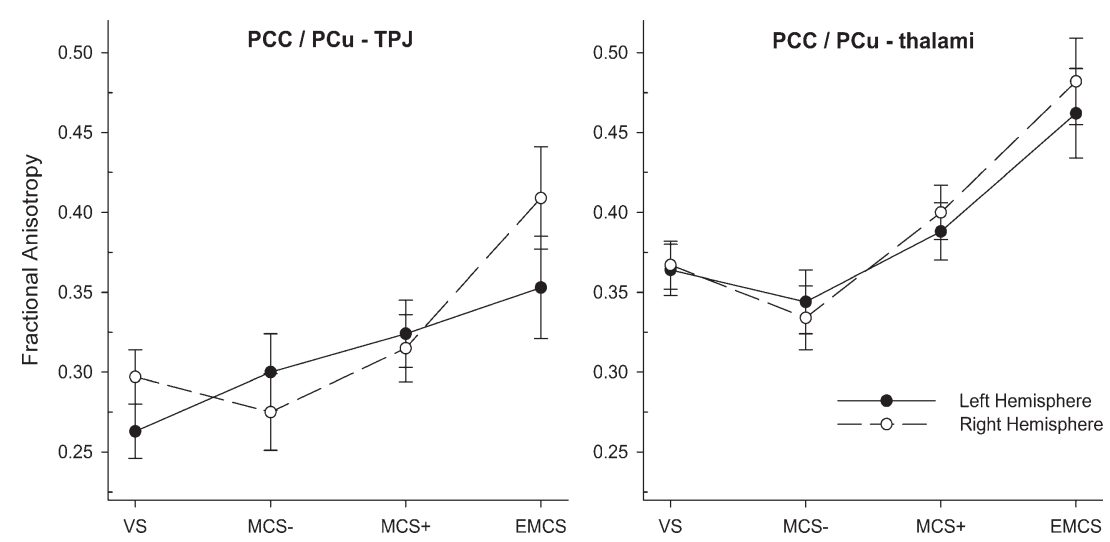

FIGURE 3: Relationship between structural integrity and clinical severity. Structural integrity of the tracts connecting the posterior cingulate cortex/precuneus with the temporoparietal junctions (on the left) and with the thalami (on the right), separated according to the clinical severity of the consciousness impairment. Each point represents the estimated marginal mean calculated by 1 -way repeated measures analysis of covariance (see text for further details). Vertical bars indicate standard errors. There was a significant effect of the amount of behavioral evidence for consciousness for both groups of tracts. There was no significant effect of hemisphere or interaction between group and hemisphere. EMCS = emerging from a minimally conscious state; MCS = minimally conscious state; $\mathrm{PCC} / \mathrm{PCu}=$ posterior cingulate cortex/precuneus; TPJ = temporoparietal junctions; VS = vegetative state.

thalamus, mainly involving the thalamic radiations, internal capsule, and corona radiata.

Although they only included cortical regions in their tractography analysis, Liao et $\mathrm{al}^{22}$ also identified these paths as secondary fibers in the DMN, suggesting possible structural connections with the thalamus. The results of the current study are consistent with this suggestion, demonstrating structural connectivity between the thalamus and all of the cortical nodes of the DMN. It is widely known that the thalamus is a relay center for the majority of cortical fiber projections. Although thalamocortical connections have been characterized in great detail for nonhuman primates and other species, ${ }^{23,24}$ they are still somewhat poorly understood in humans. Diffusion tensor imaging (DTI) tractography has recently allowed the main patterns of connectivity between the thalamus and large major cortical regions to be investigated in vivo, including prefrontal, parietal/occipital, motor/premotor, somatosensory, and temporal cortices in normal adults, ${ }^{25,26}$ but to our knowledge this is the first tractography description of the specific structural pathways that connect the thalami with the cortical areas of the DMN in humans. Our findings are consistent with demonstrated anatomical connections in the macaque. ${ }^{24}$

\section{Structural Connectivity Impairments in DOC}

Both corticocortical and thalamocortical pathways were reliably identified in most of the participants in this study. When patients were compared with healthy volunteers, they showed a reduction in the structural integrity of all of the corticocortical fibers, as well as the fibers that connect $\mathrm{PCC} / \mathrm{PCu}$ with thalami. These results are concordant with previous positron emission tomography and functional MRI studies that have shown significant metabolic reductions and functional disconnections in areas related to the DMN, as well as the thalami, in VS and MCS patients, ${ }^{1-3,5}$ and suggest a possible neuropathological substrate for that functional impairment. In vivo signs of widespread white matter damage have been previously described in DOC patients, ${ }^{12,27}$ in agreement with neuropathological studies, ${ }^{28,29}$ as well as other DTI studies of severely brain-injured patients. ${ }^{30}$ Importantly, the impairment in the integrity of the DMN revealed in this study was above and beyond the widespread white matter damage observed, suggesting a crucial role for this network, or at least, for the white matter fasciculi that connect its different cortical and subcortical nodes, in the structural bases of the DOC.

\section{Structural Connectivity and the Severity of DOC} Vanhaudenhuyse et $\mathrm{al}^{5}$ recently published the first reported correlation between functional connectivity in the DMN and behavioral indices of consciousness, leading those authors to suggest that measures of resting brain function provide a useful additional tool in the clinical assessment of DOC patients. Although the practical advantages (ie, availability, ease of acquisition, etc) of such an approach over standard functional MRI paradigms in clinical settings are undoubted, the exact meaning of a functional impairment in this network in relation to the specific brain damage in these patients was previously unclear. The results reported here complement these functional data by demonstrating a direct relationship between behavioral evidence of consciousness in these patients and structural damage to the fibers connecting the posterior regions of the DMN (ie, PCC/PCu 
and TPJ), as well as the PCC/PCu with the thalami. Importantly, damage to those connections was also correlated with the outcome of a standardized tool for the behavioral assessment of awareness, the CRS-R. However, caution should be taken when interpreting this correlation, as the CRS-R total score was not developed to differentiate between levels of consciousness; rather, it is the diagnosis from its subscales that does so. The PCC/PCu is among the more interconnected cortical areas of the brain and, along with the TPJ, is thought to play a central role in mediating activity within the default mode network, ${ }^{31}$ showing more metabolic activity at rest than any other area within the cerebral cortex. ${ }^{32}$ It is noteworthy that in the study described above, ${ }^{5}$ the only region that showed differences in functional connectivity between MCS and unconscious patients was the PCC/PCu.

The importance of the thalamus in DOC has been demonstrated previously in both neuropathological ${ }^{33}$ and neuroimaging studies. ${ }^{34}$ The crucial role of $\mathrm{PCC} / \mathrm{PCu}-$ TPJ and PCC/PCu-thalami connections suggested by the current results are in accordance with a variety of previous reports in the literature. For example, increased right-left anisotropy in medial posterior regions, comprising fibers contiguous with the splenium, was found to accompany the recovery of consciousness in a patient after 19 years in a MCS. ${ }^{35}$ Metabolic impairments in the $\mathrm{PCC} / \mathrm{PCu}$ have also been related to abnormal hyperactivation in the ascending reticular activating system in VS patients who showed a functional disconnection between the 2 regions thought to be structurally connected via the thalami. ${ }^{36}$ Finally, Boly et $\mathrm{al}^{4}$ demonstrated that, although some VS patients may retain preserved corticocortical functional connectivity in the DMN, they do not retain functional connectivity between $\mathrm{PCC} / \mathrm{PCu}$ and thalami. To our knowledge, no study has reported functional connectivity between the cortical regions of the DMN and the thalamus in VS patients, which is entirely consistent with the specific structural impairment of this pathway in this patient group reported here.

\section{Subcategorizing the MCS}

A rather serendipitous finding in the current study was the presence of structural differences between MCSand MCS + patient groups. The term MCS was first proposed in 2002 to describe patients who, although not meeting the diagnostic criteria for VS, were still not considered to be fully conscious. ${ }^{7}$ In subsequent years, evidence to support the importance of this distinction has accumulated in the scientific literature, with a vast number of studies now showing both functional and structural differences between VS and MCS groups. ${ }^{12,34,37-39}$ However, most of these studies have also stressed that, as a group, MCS patients are extremely heterogenous. To account for this, Bruno et $\mathrm{al}^{14}$ have recently proposed 2 subcategories of MCS patients, MCS - and MCS+, in order to better characterize the behavioral signs of consciousness exhibited by specific individuals within the broader group. Nevertheless, the distinction between these 2 categories is purely behavioral, based on the level of complexity of the patients' exhibited responses (ie, just nonreflex movements versus more complex behaviors such as command following), and to date no evidence of distinct underlying functional or structural features had been described in the literature.

In this study, significant differences between these 2 subgroups of patients were observed in terms of the structural integrity of the fibers connecting $\mathrm{PCC} / \mathrm{PCu}$ and thalami. This provides the first structural support for the subcategorization of MCS patients into MCS+ and MCS-. Although it is known that MCS patients have a greater potential for a favorable outcome than VS patients, ${ }^{40}$ the differential prognostic implications of MCS- versus MCS+ are still unknown. The evidence presented here for structural differences between these 2 groups should encourage further investigation into this matter.

Due to the relative rarity of DOC patients, it was necessary to combine data from 3 separate assessment centers to form our cohort. We addressed the potential impact of this factor in our analyses; however, for a full discussion of potential limitations please see the Supplementary Materials.

\section{Conclusions}

The present findings identify a specific structural impairment in the DMN in DOC patients. In the context of the previously reported functional disconnections in this network, these results suggest a critical role for the DMN in the genesis of awareness and in the neural bases of its disorders. Although nonspecific damage to this network is related to impaired consciousness (as evidenced by behavioral assessment), the specific level of this impairment appears to be primarily related to the connectivity between posterior areas of the DMN, and between these areas and the thalami. It is reasonable to conclude that structural disruptions in the fibers connecting 2 areas will also lead to a loss of, or reduction in, the functional connectivity between these regions. Therefore, the results reported here may explain why DOC patients exhibit specific functional impairments in this network.

\section{Acknowledgment}

This research was supported by generous funding from the Spanish Ministries of Science and Innovation 
(SAF2007-66077; C.J.) and Education (AP2006-00862; D.F.-E.); Medical Research Council (U.1055.01.002.00001.01; J.D.P., A.M.O.), James S. McDonnell Foundation (D.F.-E., A.S., J.D.P., A.M.O., S.L.), Canada Excellence Research Chairs Program (D.F.-E., D.C., A.M.O.), European Commission (Deployment of Brain-Computer Interfaces for the Detection of Consciousness in Nonresponsive Patients; and Consciousness: A Transdisciplinary, Integrated Approach), Fonds de la Recherche Scientifique, Mind Science Foundation, Belgian French-Speaking Community Concerted Research Action University Hospital of Liège, University of Liège, European Space Agency (S.L.) and Gates Cambridge Trust (V.N.). Medical Research Council Program Grant (Acute brain injury: heterogeneity of mechanisms, therapeutic targets and outcome effects [G9439390 ID 65883]), the UK National Institute of Health Research Biomedical Research Centre at Cambridge, and the Technology Platform funding provided by the UK Department of Health.

We thank Dr M. Coleman, Dr M. Bernabeu, and M.-A. Bruno for patient admission and assessment at Addenbrooke's Hospital (Cambridge), Hospital Clinic (Barcelona), and University Hospital of Liège, respectively.

\section{Potential Conflicts of Interest}

C.J.: grants/grants pending, Ministry of Science (Spain). V.N.: employment, NIHR Academic Clinical Fellow; grants/grants pending, ESICM research grant; D.K.M.: board membership, European Brain Injury Consortium, Intensive Care National Audit and Research Centre (London); consultancy, Solvay, GlaxoSmithKline, Brainscope, Ornim Medical, Shire Medical, Neurovive; employment, University of Cambridge, Addenbrooke's Hospital (Cambridge); speaking fees, GlaxoSmithKline; patents/ patents pending, patent registered for a new positron emission tomography ligand for assessing mitochondrial function; royalties, from Cambridge University press as coeditor of Textbook of Neuroanaesthesia and Critical Care.

\section{References}

1. Cauda F, Micon BM, Sacco K, et al. Disrupted intrinsic functional connectivity in the vegetative state. J Neurol Neurosurg Psychiatry 2009;80:429-431.

2. Laureys S, Goldman S, Phillips C, et al. Impaired effective cortical connectivity in vegetative state: preliminary investigation using PET. Neuroimage 1999;9:377-382.

3. Soddu A, Vanhaudenhuyse A, Bahri MA, et al. Identifying the default-mode component in spatial IC analyses of patients with disorders of consciousness. Hum Brain Mapp 2012;33:778-796.

4. Boly M, Tshibanda L, Vanhaudenhuyse A, et al. Functional connectivity in the default network during resting state is preserved in a vegetative but not in a brain dead patient. Hum Brain Mapp 2009;30:2393-2400.
5. Vanhaudenhuyse A, Noirhomme Q, Tshibanda LJF, et al. Default network connectivity reflects the level of consciousness in noncommunicative brain-damaged patients. Brain 2010;133:161-171.

6. Royal College of Physicians Working Party. The vegetative state: guidance on diagnosis and management. Clin Med 2003;3: 249-254.

7. Giacino JT, Ashwal S, Childs N, et al. The minimally conscious state: definition and diagnostic criteria. Neurology 2002;58: 349-353

8. Laureys S. The neural correlate of (un)awareness: lessons from the vegetative state. Trends Cogn Sci 2005;9:556-559.

9. Damoiseaux JS, Greicius MD. Greater than the sum of its parts: a review of studies combining structural connectivity and restingstate functional connectivity. Brain Struct Funct 2009;213:525-533.

10. Fernández-Espejo D, Junque C, Cruse D, et al. Combination of diffusion tensor and functional magnetic resonance imaging during recovery from the vegetative state. BMC Neurol 2010;10:77.

11. Bruno M-A, Fernández-Espejo D, Lehembre R, et al. Multimodal neuroimaging in patients with disorders of consciousness showing "functional hemispherectomy." Prog Brain Res 2011;193:325-335.

12. Fernández-Espejo D, Bekinschtein T, Monti MM, et al. Diffusion weighted imaging distinguishes the vegetative state from the minimally conscious state. Neuroimage 2011;54:103-112.

13. Giacino J, Kalmar K, Whyte J. The JFK Coma Recovery Scale-Revised: measurement characteristics and diagnostic utility. Arch Phys Med Rehabil 2004;85:2020-2029.

14. Bruno M-A, Vanhaudenhuyse A, Thibaut $A$, et al. From unresponsive wakefulness to minimally conscious PLUS and functional locked-in syndromes: recent advances in our understanding of disorders of consciousness. J Neurol 2011;258:1373-1384.

15. Behrens TEJ, Johansen-Berg H, Jbabdi S, et al. Probabilistic diffusion tractography with multiple fibre orientations: what can we gain? Neuroimage 2007;34:144-155.

16. Fox MD, Snyder AZ, Vincent $J L$, et al. The human brain is intrinsically organized into dynamic, anticorrelated functional networks. Proc Natl Acad Sci U S A 2005;102:9673-9678.

17. Jenkinson M, Bannister $P$, Brady M, Smith S. Improved optimization for the robust and accurate linear registration and motion correction of brain images. Neuroimage 2002;17:825-841.

18. Mori S, van Zijl PCM. Fiber tracking: principles and strategies-a technical review. NMR Biomed 2002;15:468-480.

19. van den Heuvel M, Mandl R, Luigjes J, Hulshoff Pol H. Microstructural organization of the cingulum tract and the level of default mode functional connectivity. J Neurosci 2008;28:10844-10851.

20. Sala-Llonch R, Bosch B, Arenaza-Urquijo EM, et al. Greater default-mode network abnormalities compared to high order visual processing systems in amnestic mild cognitive impairment: an integrated multi-modal MRI study. J Alzheimers Dis 2010;22: 523-539.

21. Supekar K, Uddin LQ, Prater K, et al. Development of functional and structural connectivity within the default mode network in young children. Neuroimage 2010;52:290-301.

22. Liao W, Zhang Z, Pan Z, et al. Default mode network abnormalities in mesial temporal lobe epilepsy: a study combining fMRI and DTI. Hum Brain Mapp 2011;32:883-895.

23. Guillery RW, Sherman SM. Branched thalamic afferents: what are the messages that they relay to the cortex? Brain Res Rev 2011; 66:205-219.

24. Buckwalter JA, Parvizi J, Morecraft RJ, van Hoesen GW. Thalamic projections to the posteromedial cortex in the macaque. J Comp Neurol 2008;507:1709-1733.

25. Klein JC, Rushworth MFS, Behrens TEJ, et al. Topography of connections between human prefrontal cortex and mediodorsal thalamus studied with diffusion tractography. Neuroimage 2010;51:555-564. 
26. Zhang D, Snyder AZ, Shimony JS, et al. Noninvasive functional and structural connectivity mapping of the human thalamocortical system. Cereb Cortex 2010;20:1187-1194.

27. Newcombe VFJ, Williams GB, Scoffings D, et al. Aetiological differences in neuroanatomy of the vegetative state: insights from diffusion tensor imaging and functional implications. J Neurol Neurosurg Psychiatry 2010;81:552-561

28. Kinney HC, Samuels MA. Neuropathology of the persistent vegetative state. A review. J Neuropathol Exp Neurol 1994;53:548-558.

29. Adams JH, Jennett B, McLellan DR, et al. The neuropathology of the vegetative state after head injury. J Clin Pathol 1999;52:804-806.

30. Tollard E, Galanaud D, Perlbarg V, et al. Experience of diffusion tensor imaging and $1 \mathrm{H}$ spectroscopy for outcome prediction in severe traumatic brain injury: preliminary results. Crit Care Med 2009;37:1448-1455

31. Fransson P, Marrelec G. The precuneus/posterior cingulate cortex plays a pivotal role in the default mode network: evidence from a partial correlation network analysis. Neuroimage 2008;42:1178-1184.

32. Gusnard DA, Raichle ME. Searching for a baseline: functional imaging and the resting human brain. Nat Rev Neurosci 2001;2:685-694.

33. Maxwell WL, MacKinnon MA, Smith $\mathrm{DH}$, et al. Thalamic nuclei after human blunt head injury. J Neuropathol Exp Neurol 2006;65: 478-488.
34. Fernández-Espejo D, Junque $\mathrm{C}$, Bernabeu $\mathrm{M}$, et al. Reductions of thalamic volume and regional shape changes in the vegetative and the minimally conscious states. J Neurotrauma 2010;27: 1187-1193.

35. Voss HU, Uluç AM, Dyke JP, et al. Possible axonal regrowth in late recovery from the minimally conscious state. J Clin Invest 2006;116:2005-2011

36. Silva S, Alacoque $X$, Fourcade $O$, et al. Wakefulness and loss of awareness. Brain and brainstem interaction in the vegetative state. Neurology 2010;74:313-320

37. Di HB, Yu SM, Weng XC, et al. Cerebral response to patient's own name in the vegetative and minimally conscious states. Neu rology 2007;68:895-899.

38. Boly M, Faymonville M-E, Schnakers $C$, et al. Perception of pain in the minimally conscious state with PET activation: an observational study. Lancet Neurol 2008;7:1013-1020.

39. Boly $M$, Faymonville $M-E$, Peigneux $P$, et al. Auditory processing in severely brain injured patients: differences between the minimally conscious state and the persistent vegetative state. Arch Neurol 2004;61:233-238

40. Luauté J, Maucort-Boulch D, Tell L, et al. Long-term outcomes of chronic minimally conscious and vegetative states. Neurology 2010;75:246-252. 